\title{
Generalized stabilizability for bivariate means
}

\author{
Abdessalem Gasmi ${ }^{*}$ and Mustapha Raïssouli1,2
}

\section{"Correspondence:}

aguesmi@taibahu.edu.sa

'Department of Mathematics, Faculty of Science, Taibah University, P.O. Box 30097, Al Madinah Al Munawwarah, 41477, Kingdom of Saudi Arabia

Full list of author information is available at the end of the article

\begin{abstract}
The stabilizability concept for bivariate means has been introduced and studied in (Raïssouli in Appl. Math. E-Notes, 11:159-174, 2011). It has been proved to be a useful tool for theoretical viewpoint as well as for practical purposes (Raïssouli in Appl. Math. E-Notes, 11:159-174, 2011). In the present paper, we give an extension of the stabilizability concept, the so-called generalized stabilizability. Our paper will be completed by some applications and examples illustrating the above extension and showing the interest of this work.
\end{abstract}

\section{Introduction}

In this section, we state some basic notions about bivariate means, see [1]. A function $m:(0, \infty) \times(0, \infty) \rightarrow(0, \infty)$ is called a (bivariate) mean if

$$
\forall a, b>0 \quad \min (a, b) \leq m(a, b) \leq \max (a, b)
$$

It is clear that every mean is with positive values and reflexive, that is, $m(a, a)=a$ for all $a>0$. The maps $(a, b) \mapsto \min (a, b)$ and $(a, b) \mapsto \max (a, b)$ are (trivial) means which will be denoted by min and max, respectively. The standard examples of means are as follows [2]:

$$
\begin{aligned}
& A:=A(a, b)=\frac{a+b}{2} ; \quad G:=G(a, b)=\sqrt{a b} ; \quad H:=H(a, b)=\frac{2 a b}{a+b} ; \\
& L:=L(a, b)=\frac{b-a}{\ln b-\ln a}, \quad L(a, a)=a ; \\
& I:=I(a, b)=\frac{1}{e}\left(\frac{b^{b}}{a^{a}}\right)^{1 /(b-a)}, \quad I(a, a)=a ; \\
& S:=S(a, b)=a^{a /(a+b)} b^{b /(a+b)} ; \quad C:=C(a, b)=\frac{a^{2}+b^{2}}{a+b} ;
\end{aligned}
$$

they are known in the literature as the arithmetic, geometric, harmonic, logarithmic, identric, weighted geometric and contra-harmonic means, respectively. It is well known that

$$
H(a, b) \leq G(a, b) \leq L(a, b) \leq I(a, b) \leq A(a, b) \leq S(a, b) \leq C(a, b)
$$

for all $a, b>0$ with equalities if and only if $a=b$.

A mean $m$ is symmetric if $m(a, b)=m(b, a)$ and homogeneous if $m(t a, t b)=t m(a, b)$ for all $a, b, t>0$. The above means are all symmetric and homogeneous. A mean $m$ is called monotone if $(a, b) \mapsto m(a, b)$ is increasing in $a$ and in $b$, that is, $a_{1} \leq a_{2}$ and $b_{1} \leq b_{2}$ imply

(c) 2013 Gasmi and Raïssouli; licensee Springer. This is an Open Access article distributed under the terms of the Creative Commons Attribution License (http://creativecommons.org/licenses/by/2.0), which permits unrestricted use, distribution, and reproduction in any medium, provided the original work is properly cited. 
$m\left(a_{1}, b\right) \leq m\left(a_{2}, b\right)$ and $m\left(a, b_{1}\right) \leq m\left(a, b_{2}\right)$. It is not hard to check that the means $A, G, H$, $L, I$ are monotone but $S$ and $C$ are not.

Let $m$ be a homogeneous mean. Writing $m(a, b)=a m(1, b / a)$, we then associate to $m$ a unique positive function $f$ defined by $f(x)=m(1, x)$ for all $x>0$. The function $f$ will be called an associated function to the mean $m$, or we simply say that $f$ corresponds to the mean $m$. It follows that $f$ corresponds to a homogeneous mean if and only if $\min (1, x) \leq$ $f(x) \leq \max (1, x)$ for every $x>0$. Clearly, $f(1)=1$ and if, moreover, $m$ is symmetric, then $f(x)=x f(1 / x)$ for each $x>0$. It is obvious that a mean $m$ is monotone if and only if its associated function is increasing. For example, and as pointed in [1], the contraharmonic mean $C$ is not monotone because its associated function $f(x)=\left(x^{2}+1\right) /(x+1)$ satisfies $(x+1)^{2} f^{\prime}(x)=x^{2}+2 x-1$, and it is easy to see that $f$ is not increasing for all $x>0$, but only for $x>\sqrt{2}-1$.

In the literature, there are some families of means, called power means, which include the above familiar means. Let $p$ and $q$ be two real numbers. The Stolarsky mean $E_{p, q}$ of order $(p, q)$ is defined by [3]

$$
E_{p, q}:=E_{p, q}(a, b)= \begin{cases}\left(\frac{p}{q} \frac{b^{q}-a^{q}}{b^{p}-a^{p}}\right)^{1 /(q-p)} & \text { if } p q(p-q) \neq 0, \\ \exp \left(-\frac{1}{p}+\frac{a^{p} \ln a-b^{p} \ln b}{a^{p}-b^{p}}\right) & \text { if } p=q \neq 0, \\ \left(\frac{1}{p} \frac{b^{p}-a^{p}}{\ln b-\ln a}\right)^{1 / p} & \text { if } p \neq 0, q=0, \\ \sqrt{a b} & \text { if } p=q=0\end{cases}
$$

with $E_{p, q}(a, a)=a$.

It is understood that this family of means includes some interesting cases as well:

- The power binomial mean

$$
\left\{\begin{array}{l}
E_{p, 2 p}(a, b):=B_{p}(a, b)=B_{p}=\left(\frac{a^{p}+b^{p}}{2}\right)^{1 / p}, \\
B_{-\infty}=\min , \quad B_{-1}=H, \quad B_{1}=A, \quad B_{0}:=\lim _{p \rightarrow 0} B_{p}=G, \quad B_{\infty}=\max .
\end{array}\right.
$$

- The power logarithmic mean

$$
\begin{cases}E_{1, p+1}:=L_{p}(a, b)=L_{p}=\left(\frac{a^{p+1}-b^{p+1}}{(p+1)(a-b)}\right)^{1 / p}, & L_{p}(a, a)=a, \\ L_{-\infty}=\min , \quad L_{-2}=G, \quad L_{-1}=L, & L_{0}=I, \quad L_{1}=A, \quad L_{\infty}=\max .\end{cases}
$$

It is easy to see that $E_{p, q}$ is homogeneous and symmetric for all fixed real numbers $p$ and $q$. Further, it is clear that $E_{p, q}$ is symmetric in $p$ and $q$, and it is well known that $E_{p, q}$ is strictly increasing in both $p$ and $q$. In particular the power means $B_{p}$ and $L_{p}$ are strictly increasing in $p$.

The reminder of this paper is organized as follows. Section 2 displays a class of means, the so-called parameterized means that will be needed later. Section 3 is focused on introducing a new definition that includes a lot of standard means. Section 4 is devoted to extending the resultant mean-map, introduced by the second author in an earlier paper for means, to positive maps in the aim to state conveniently the generalized stabilizability in Section 5. This latter concept is then applied to standard (power) means. In Section 6, application to mean inequalities is investigated and interesting examples are discussed. 


\section{Parameterized bivariate means}

This section is focused on stating a special class of means depending on a real parameter, the so-called parameterized means, defined as follows.

Definition 2.1 Let $\left(m_{t}\right)_{0 \leq t \leq 1}$ be a family of maps with $m_{t}:(0, \infty) \times(0, \infty) \rightarrow(0, \infty)$ for fixed $t \in[0,1]$. We say that $\left(m_{t}\right)$ is a parameterized (or weighted) mean if the following assertions are satisfied:

(i) $m_{t}$ is a mean for all fixed $t \in[0,1]$,

(ii) For all $a, b>0, m_{0}(a, b)=a$ and $m_{1}(a, b)=b$ or, $m_{0}(a, b)=b$ and $m_{1}(a, b)=a$,

(iii) $m_{t}(a, b)=m_{1-t}(b, a)$ for all $a, b>0$ and $t \in[0,1]$.

From the above definition, we deduce that $m_{1 / 2}$ is a symmetric mean which we call the associated (symmetric) mean of the parameterized mean $\left(m_{t}\right)$. The following

$$
A_{t}(a, b)=(1-t) a+t b, \quad G_{t}(a, b)=a^{1-t} b^{t}, \quad H_{t}(a, b)=\left((1-t) a^{-1}+t b^{-1}\right)^{-1}
$$

are known in the literature as the parameterized arithmetic, geometric and harmonic means, respectively. For all $t \in[0,1], A_{t}, G_{t}$ and $H_{t}$ are homogeneous monotone, but not symmetric unless $t=1 / 2$. Clearly, $A_{1 / 2}=A, G_{1 / 2}=G$ and $H_{1 / 2}=H$ which are the associated symmetric means of $\left(A_{t}\right),\left(G_{t}\right)$ and $\left(H_{t}\right)$ respectively. The parameterized weighted geometric and contra-harmonic means are given by

$$
S_{t}(a, b)=\left(a^{t a} b^{(1-t) b}\right)^{1 / A_{t}}, \quad C_{t}(a, b)=\frac{A_{t}\left(a^{2}, b^{2}\right)}{A_{t}(a, b)} .
$$

It is easy to see that $S_{1 / 2}=S$ and $C_{1 / 2}=C$.

The parameterized logarithmic mean was introduced in [4] as follows:

$$
L_{t}(a, b)=\prod_{n=1}^{\infty} A_{t}\left(a^{1 / 2^{n}}, b^{1 / 2^{n}}\right):=\prod_{n=1}^{\infty}\left((1-t) a^{1 / 2^{n}}+t b^{1 / 2^{n}}\right)
$$

while the parameterized identric mean can be inspired from [1] as well

$$
I_{t}(a, b)=\prod_{n=1}^{\infty} S_{t}\left(a^{1 / 2^{n}}, b^{1 / 2^{n}}\right)
$$

It is proved in [5] that $L_{1 / 2}=L$ and in [1] that $I_{1 / 2}=I$.

The above parameterized means satisfy the following chain of inequalities (see [1]):

$$
\begin{aligned}
\min (a, b) & \leq H_{t}(a, b) \leq G_{t}(a, b) \leq L_{t}(a, b) \leq I_{t}(a, b) \\
& \leq A_{t}(a, b) \leq S_{t}(a, b) \leq C_{t}(a, b) \leq \max (a, b),
\end{aligned}
$$

with strict inequalities if and only if $a \neq b$ and $t \in(0,1)$.

The parameterized power binomial mean can be immediately given by

$$
B_{p, t}(a, b)=\left((1-t) a^{p}+t b^{p}\right)^{1 / p},
$$


for $p \neq 0$, with $B_{0, t}(a, b)=a^{1-t} b^{t}=G_{t}(a, b)$. The parameterized Stolarsky mean $E_{p, q, t}$ can be also inspired from [1] by setting

$$
E_{p, q, t}(a, b)=\prod_{n=1}^{\infty} G_{p, q, t}\left(a^{1 / 2^{n}}, b^{1 / 2^{n}}\right)
$$

where $G_{p, q, t}$ refers to the parameterized Gini mean defined through

$$
G_{p, q, t}(a, b):= \begin{cases}\left(\frac{(1-t) a^{q}+t b^{q}}{\left.(1-t) a^{p}+t b\right)^{1 /(q-p)}}\right. & \text { if } p \neq q, \\ \exp \left(\frac{(-1) a^{p} \ln a+t b^{p} \ln b}{(1-t) a^{p}+t b^{p}}\right) & \text { if } p=q \neq 0, \\ a^{1-t} b^{t} & \text { if } p=q=0 .\end{cases}
$$

In particular, the parameterized power logarithmic mean is given by

$$
L_{p, t}(a, b)=\prod_{n=1}^{\infty} M_{p, t}\left(a^{1 / 2^{n}}, b^{1 / 2^{n}}\right),
$$

where $M_{p, t}$ stands for the parameterized mean defined through

$$
M_{p, t}(a, b)=\left(\frac{(1-t) a^{p+1}+t b^{p+1}}{(1-t) a+t b}\right)^{1 / p} .
$$

Let $(\alpha, \beta)$ be a (closed or open) interval of $\mathbb{R}$. Since there exists a homeomorphism between $(0,1)$ and $(\alpha, \beta)$, we then can define a parameterized mean $\left(m_{t}\right)$ indexed by $t \in(\alpha, \beta)$ in the following sense:

(j) $m_{t}$ is a mean for all fixed $t \in(\alpha, \beta)$,

(jj) for all $a, b>0, m_{\alpha}(a, b)=a$ and $m_{\beta}(a, b)=b$ or, $m_{\alpha}(a, b)=b$ and $m_{\beta}(a, b)=a$,

(jij) $m_{t}(a, b)=m_{\alpha+\beta-t}(b, a)$ for all $a, b>0$ and $t \in[\alpha, \beta]$.

The associated symmetric mean of $\left(m_{t}\right)_{\alpha \leq t \leq \beta}$ is $m_{(\alpha+\beta) / 2}$. Let us observe the next example explaining this latter situation.

Example 2.1 Let $p$ be a fixed real number. For $a, b>0$ and $0 \leq t \leq \pi / 2$, we set

$$
m_{p, t}(a, b)=\left(a^{p} \cos ^{2} t+b^{p} \sin ^{2} t\right)^{1 / p} .
$$

It is easy to verify that $\left(m_{p, t}\right)_{0 \leq t \leq \pi / 2}$ satisfies $(\mathrm{j})$,(jj) and (jjj), then it is a parameterized mean. The associated symmetric mean $m_{p, \pi / 4}$ is the power (binomial) mean $B_{p}$.

By the simple transformation $s=(1-t) \alpha+t \beta$, it is always possible to reduce $\left(m_{s}\right)_{\alpha \leq s \leq \beta}$ into $\left(m_{t}\right)_{0 \leq t \leq 1}$. Henceforth, when we consider a parameterized mean $\left(m_{t}\right)$, it will be indexed by $t \in[0,1]$.

\section{Class of $(\Phi, \phi)_{m}$-means}

Let $\left(m_{t}\right)_{0 \leq t \leq 1}$ be a given parameterized mean. Assume that $m_{t}$ is continuous with respect to the variable $t \in[0,1]$, then it is easy to sketch that the maps

$$
E(a, b)=\int_{0}^{1} m_{t}(a, b) d t \quad \text { and } \quad F(a, b)=\exp \int_{0}^{1} \ln \left(m_{t}(a, b)\right) d t
$$

define two bivariate symmetric means. The following examples illustrate this situation. 
Example 3.1 It is well known that

$$
\begin{aligned}
L(a, b) & =\int_{0}^{1} a^{1-t} b^{t} d t=\int_{0}^{1} G_{t}(a, b) d t \\
I(a, b) & =\exp \int_{0}^{1} \ln ((1-t) a+t b) d t \\
& =\exp \int_{0}^{1} \ln A_{t}(a, b) d t
\end{aligned}
$$

and

$$
L^{*}(a, b)=\int_{0}^{1} H_{t}(a, b) d t
$$

where

$$
L^{*}:=L^{*}(a, b)=\left(L\left(a^{-1}, b^{-1}\right)\right)^{-1}
$$

refers to the dual of the logarithmic mean $L$.

Example 3.2 The following

$$
F(a, b)=\exp \int_{0}^{1} \ln L_{t}(a, b) d t
$$

defines a symmetric mean. It is proved in [4] that

$$
F(a, b)=\prod_{n=1}^{\infty} I\left(a^{1 / 2^{n}}, b^{1 / 2^{n}}\right)
$$

See also [1] for a general approach including the above expansion.

Example 3.3 For $a, b>0$ and $0 \leq t \leq 1$, we set

$$
m_{t}(a, b)=\sqrt{a^{2} \cos ^{2} t \pi / 2+b^{2} \sin ^{2} t \pi / 2} .
$$

It is easy to verify that $\left(m_{t}\right)_{0 \leq t \leq 1}$ defines a parameterized mean. Then the expression

$$
T(a, b)=\int_{0}^{1} \sqrt{a^{2} \cos ^{2} t \pi / 2+b^{2} \sin ^{2} t \pi / 2} d t
$$

defines a symmetric mean. Taking $\theta=t \pi / 2$, we see that $T(a, b)$ is the Toader mean introduced in [6].

In what follows we are interested in extending the situation of the two above examples. We may then state the next definition. 
Definition 3.1 Let $m:=\left(m_{t}\right)_{0 \leq t \leq 1}$ be a parameterized mean, continuous with respect to $t \in[0,1]$. A mean $M$, for which there exist two continuous strictly monotonic functions $\Phi:(0, \infty) \rightarrow \Phi((0, \infty))$ and $\phi:(0, \infty) \rightarrow(0, \infty)$ such that

$$
\forall a, b>0 \quad \Phi(M(a, b))=\int_{0}^{1} \Phi \circ \phi^{-1} \circ m_{t}(\phi(a), \phi(b)) d t,
$$

will be called a $(\Phi, \phi)_{m}$-mean.

We explicitly notice that, by virtue of Definition 2.1(iii) and with the change of variable $t=1-s$ in (3.6), we deduce that every $(\Phi, \phi)_{m}$-mean is symmetric. For this, we need to assume that the involved means, in the announced theoretical results, are symmetric.

If in the above definition we have $\Phi=\phi$, then (3.6) becomes

$$
\Phi(M(a, b))=\int_{0}^{1} m_{t}(\Phi(a), \Phi(b)) d t .
$$

In particular, with $m_{t}=A_{t}$ we obtain

$$
\Phi(M(a, b))=A(\Phi(a), \Phi(b))
$$

as a special class of means known in the literature as quasi-arithmetic means. The power binomial mean $B_{p}$ is quasi-arithmetic with $\Phi(x)=x^{p}$ for $p \neq 0$ and $\Phi(x)=\ln x$ for $p=0$. In summary, the means $A, G, H$ are included in the above definition. Following (3.2) and (3.3) the means $L$ and $I$ are also included in the above. More generally, the power mean $E_{p, q}$, for all real numbers $p$ and $q$, can be also obtained as a particular case of the above definition when convenient functions $\Phi$ and $\phi$ are chosen. Let us observe the next example explaining this latter situation.

Example 3.4 Let $p$ and $q$ be two fixed real numbers. Assume that $p \neq 0$ and $p \neq q$.

(i) Let us choose $m=\left(m_{t}\right)=\left(A_{t}\right)$ and $\phi(x)=x^{p}, \Phi(x)=x^{q-p}$. In this case the symmetric mean obtained through (3.6) is given by

$$
(M(a, b))^{q-p}=\int_{0}^{1}\left((1-t) a^{p}+t b^{p}\right)^{(q-p) / p} d t .
$$

A simple computation shows that $M(a, b)$ coincides with $E_{p, q}(a, b)$. This, with Definition 3.1, means that the Stolarsky mean $E_{p, q}$ is a $(\Phi, \phi)_{m}$-mean with $\Phi, \phi$ and $m$ previously defined.

(ii) We can obtain the same as in (i) by choosing $m=\left(m_{t}\right)=\left(B_{p, t}\right)$ and $\phi(x)=x$, $\Phi(x)=x^{q-p}$ and so $E_{p, q}$ is a $(\Phi, i d)_{m}$-mean.

For $p=0$ or $p=q$, we left the reader to choose the convenient functions $\phi$ and $\Phi$ for $E_{0, q}$ and $E_{p, p}$ in a similar manner as a previous one.

Remark 3.1 Formula (3.6) in the particular case $m=\left(m_{t}\right)=\left(A_{t}\right)$ was considered by Toader and Sándor [7] in the aim to obtain some mean-inequalities when convenient hypotheses on $\phi$ and $\Phi$ are assumed. Here, we consider (3.6) in its general form in the aim to introduce some mean-concepts and investigate some related applications. 


\section{Resultant functional-map}

In [8], the author defined the resultant mean-map concept for bivariate means as a good tool for introducing the stability and stabilizability notions. This concept can be extended for functionals instead of means as well.

Definition 4.1 Let $f, g, h:(0, \infty) \times(0, \infty) \rightarrow(0, \infty)$ be three given functions. For $a, b>0$, define

$$
\mathcal{R}(f, g, h)(a, b)=f(g(a, h(a, b)), g(h(b, a), b)),
$$

which we call the resultant functional-map of $f, g$ and $h$.

We explicitly notice that $\mathcal{R}$ is a map with three functionals variables $f, g, h$ (which justifies the chosen terminology), while $\mathcal{R}(f, g, h)$ is a functional with two positive real variables $a, b$. If $f, g, h$ are bivariate symmetric means, the above definition coincides with that introduced in [8]. In our next study, we will be restricted by the case where the functionals $f$ and $h$ are bivariate symmetric means, while $g$ is such that $g=\Phi \circ m$, where $m$ is a symmetric mean and $\Phi:(0, \infty) \rightarrow \Phi((0, \infty))$ is a continuous strictly monotonic function. For the sake of simplicity, we write $\Phi(m)$ instead of $\Phi \circ m$. For all three given means $m_{1}, m_{2}$, $m_{3}$, we also use the following notation:

$$
\mathcal{R}_{\Phi}\left(m_{1}, m_{2}, m_{3}\right):=\Phi^{-1} \circ \mathcal{R}\left(m_{1}, \Phi\left(m_{2}\right), m_{3}\right) .
$$

With this, the next result may be stated.

Proposition 4.1 Let $m_{1}, m_{2}, m_{3}$ be three symmetric means with $m_{1}$ and $m_{2}$ monotone, and let $\Phi:(0, \infty) \rightarrow \Phi((0, \infty))$ be a continuous strictly monotonic function. Then the following assertions are satisfied:

(i) $\mathcal{R}_{\Phi}\left(m_{1}, m_{2}, m_{3}\right)$ is a mean.

(ii) If $m_{1}^{\prime}, m_{2}^{\prime}, m_{3}^{\prime}$ are three means such that $m_{1} \leq m_{1}^{\prime}, m_{2} \leq m_{2}^{\prime}, m_{3} \leq m_{3}^{\prime}$, then we have

$$
\mathcal{R}_{\Phi}\left(m_{1}, m_{2}, m_{3}\right) \leq \mathcal{R}_{\Phi}\left(m_{1}^{\prime}, m_{2}^{\prime}, m_{3}^{\prime}\right)
$$

Proof We limit our attention in this proof to $\Phi$ strictly increasing, since the case of $\Phi$ strictly decreasing can be stated in a similar manner.

(i) Since $m_{2}$ is a mean, then by definition, for all $a, b>0$, we have

$$
\min (a, b) \leq m_{2}\left(a, m_{3}(a, b)\right) \leq \max (a, b)
$$

and

$$
\min (a, b) \leq m_{2}\left(m_{3}(a, b), b\right) \leq \max (a, b) .
$$

This, with the fact that $\Phi$ is increasing, yields

$$
\min (\Phi(a), \Phi(b)) \leq \Phi\left(m_{2}\right)\left(a, m_{3}(a, b)\right) \leq \max (\Phi(a), \Phi(b)),
$$


and

$$
\min (\Phi(a), \Phi(b)) \leq \Phi\left(m_{2}\right)\left(m_{3}(a, b), b\right) \leq \max (\Phi(a), \Phi(b))
$$

By the monotonicity of $m_{1}$, we get

$$
\min (\Phi(a), \Phi(b)) \leq m_{1}\left(\Phi\left(m_{2}\right)\left(a, m_{3}(a, b)\right), \Phi\left(m_{2}\right)\left(m_{3}(a, b), b\right)\right) \leq \max (\Phi(a), \Phi(b)) .
$$

Using the fact that $\Phi^{-1}$ is increasing too, we obtain

$$
\min (a, b) \leq \mathcal{R}_{\Phi}\left(m_{1}, m_{2}, m_{3}\right)(a, b) \leq \max (a, b),
$$

which means that $\mathcal{R}_{\Phi}\left(m_{1}, m_{2}, m_{3}\right)$ is a mean.

(ii) Since $m_{3}(a, b) \leq m_{3}^{\prime}(a, b)$, we deduce, with the monotonicity of $m_{2}$,

$$
m_{2}\left(a, m_{3}(a, b)\right) \leq m_{2}\left(a, m_{3}^{\prime}(a, b)\right)
$$

It follows that

$$
m_{2}\left(a, m_{3}(a, b)\right) \leq m_{2}^{\prime}\left(a, m_{3}^{\prime}(a, b)\right)
$$

and

$$
m_{2}\left(m_{3}(a, b), b\right) \leq m_{2}^{\prime}\left(m_{3}^{\prime}(a, b), b\right)
$$

The increased monotonicity of $\Phi$ implies that

$$
\Phi\left(m_{2}\right)\left(a, m_{3}(a, b)\right) \leq \Phi\left(m_{2}^{\prime}\right)\left(a, m_{3}^{\prime}(a, b)\right)
$$

and so

$$
\Phi\left(m_{2}\right)\left(m_{3}(a, b), b\right) \leq \Phi\left(m_{2}^{\prime}\right)\left(m_{3}^{\prime}(a, b), b\right) .
$$

Now, from $m_{1} \leq m_{1}^{\prime}$ with the monotonicity of $m_{1}$, we infer that

$$
\begin{aligned}
& m_{1}\left(\Phi\left(m_{2}\right)\left(a, m_{3}(a, b)\right), \Phi\left(m_{2}\right)\left(m_{3}(a, b), b\right)\right) \\
& \quad \leq m_{1}^{\prime}\left(\Phi\left(m_{2}^{\prime}\right)\left(a, m_{3}^{\prime}(a, b)\right), \Phi\left(m_{2}^{\prime}\right)\left(m_{3}^{\prime}(a, b), b\right)\right) .
\end{aligned}
$$

Finally, using the fact that $\Phi^{-1}$ is increasing with the definition of $\mathcal{R}_{\Phi}$, we obtain the desired result, which completes the proof.

Now, let us observe the following example illustrating the above.

Example 4.1 Let $\Phi(x)=2 x-1$. An elementary computation gives $\mathcal{R}_{\Phi}(A, A, A)=A$. Note that here the function $\Phi$ is the inverse of the associated function of the homogeneous mean $A$, i.e., $\Phi^{-1}(x)=A(1, x)$. Similarly, we verify that $\mathcal{R}_{\Phi}(G, G, G)=G$ with $\Phi(x)=x^{2}$, and an analogous remark as the previous one holds. 
Example 4.2 (1) Let $\Phi(x)=1 / x, x>0$. Then relationships (3.4) and (3.5) can be summarized by $\mathcal{R}_{\Phi}(A, L, A)=L$.

(2) Let $\Phi(x)=\ln x, x>0$. Expression (3.3) can be written as $\mathcal{R}_{\Phi}(A, I, A)=I$.

These relationships, and a lot of others, will be seen and interpreted in a general point of view. See section below.

Now, let us state the next example which will be needed later. We omit the computation details for the reader.

Example 4.3 Take $\Phi(x)=1 / x, x>0$. It is not hard to establish that

$$
\begin{aligned}
& \mathcal{R}_{\Phi}(A, G, A)=G\left(\frac{2 A}{A+G}\right)^{1 / 2}, \\
& \mathcal{R}_{\Phi}\left(A, \frac{A+G}{2}, A\right)=\frac{1}{2} \frac{\left(\frac{A+G}{2}+\left(\frac{A^{2}+A G}{2}\right)^{1 / 2}\right)^{2}}{A+\left(\frac{A^{2}+A G}{2}\right)^{1 / 2}} .
\end{aligned}
$$

\section{Generalized stabilizability}

As already pointed before, this section will be focused on presenting an extension of the stabilizability concept already introduced in [8]. We then may state the following definition.

Definition 5.1 Let $M$ be a given symmetric mean. Assume that there exist two nontrivial symmetric means $m_{1}, m_{2}$ and a continuous strictly monotonic function $\Phi:(0, \infty) \rightarrow$ $\Phi((0, \infty))$ such that

$$
\mathcal{R}_{\Phi}\left(m_{1}, M, m_{2}\right)=M
$$

Then we say that $M$ is $\left(m_{1}, m_{2}\right)_{\Phi}$-stabilizable.

If $m_{1}=m_{2}=M$, that is, $\mathcal{R}_{\Phi}(M, M, M)=M$, we then say $M$ is $\Phi$-stable.

In other words, $M$ is $\left(m_{1}, m_{2}\right)_{\phi}$-stabilizable if and only if $M$ is a mean-fixed point of the map $m \mapsto \mathcal{R}_{\Phi}\left(m_{1}, m, m_{2}\right)$.

If $\Phi=I d$ the identity function, then the above definition coincides with that of stabilizability, and in this case, we simply say $M$ is $\left(m_{1}, m_{2}\right)$-stabilizable.

Now, let us observe the next example illustrating the above definition.

Example 5.1 Following Example 4.1 we can say that $A$ is $\Phi$-stable with $\Phi(x)=2 x-1$ and, $G$ is $\Phi$-stable with $\Phi(x)=x^{2}$. We left the reader to show that $H$ is $\Phi$-stable with a convenient function $\Phi$ to be defined.

Example 5.2 According to Example 4.2, the logarithmic mean $L$ is $(A, A)_{\Phi}$-stabilizable with $\Phi(x)=1 / x$, while the identric mean $I$ is $(A, A)_{\Phi}$-stabilizable with $\Phi(x)=\ln x$.

We now are in a position to state the next result which will be with interest of giving other examples of means satisfying the situation of the above definition. 
Theorem 5.1 Let $m:=\left(m_{t}\right)_{0 \leq t \leq 1}$ be a parameterized mean such that

$$
\forall a, b>0, \forall t \in[0,1] \quad m_{t}\left(a, m_{1 / 2}(a, b)\right)=m_{t / 2}(a, b) .
$$

Let $\Phi, \phi$ be as in Definition 3.1 and let us set

$$
m_{\phi}(a, b)=\phi^{-1}\left(m_{1 / 2}(\phi(a), \phi(b))\right) .
$$

Then every $(\Phi, \phi)_{m}$-mean $M$ is $\left(A, m_{\phi}\right)_{\Phi}$-stabilizable, that is, $\mathcal{R}_{\Phi}\left(A, M, m_{\phi}\right)=M$.

Proof Definition 3.1 with (5.2) yields

$$
\Phi(M)\left(a, m_{\phi}(a, b)\right)=\int_{0}^{1} \Phi \circ \phi^{-1} \circ m_{t}\left(\phi(a), m_{1 / 2}(\phi(a), \phi(b))\right) d t .
$$

Making the change of variable $u=t / 2$ in (5.3), we get

$$
\Phi(M)\left(a, m_{\phi}(a, b)\right)=2 \int_{0}^{\frac{1}{2}} \Phi \circ \phi^{-1} \circ m_{2 u}\left(\phi(a), m_{1 / 2}(\phi(a), \phi(b))\right) d u .
$$

This, with condition (5.1), yields

$$
\Phi(M)\left(a, m_{\phi}(a, b)\right)=2 \int_{0}^{\frac{1}{2}} \Phi \circ \phi^{-1} \circ m_{u}(\phi(a), \phi(b)) d u .
$$

Similarly, the change of variable $u=(t+1) / 2$ leads to the following:

$$
\begin{aligned}
\Phi(M)\left(m_{\phi}(a, b), b\right) & =\int_{0}^{1} \Phi \circ \phi^{-1} \circ m_{t}\left(m_{1 / 2}(\phi(a), \phi(b)), \phi(b)\right) d t \\
& =2 \int_{\frac{1}{2}}^{1} \Phi \circ \phi^{-1} \circ m_{2 u-1}\left(m_{1 / 2}(\phi(a), \phi(b)), \phi(b)\right) d u .
\end{aligned}
$$

According to (iii) of Definition 2.1, we can write

$$
m_{2 u-1}\left(m_{1 / 2}(\phi(a), \phi(b)), \phi(b)\right)=m_{2(1-u)}\left(\phi(b), m_{1 / 2}(\phi(b), \phi(a))\right) .
$$

Using (5.1), we obtain

$$
\Phi(M)\left(m_{\phi}(a, b), b\right)=2 \int_{\frac{1}{2}}^{1} \Phi \circ \phi^{-1} \circ m_{u}(\phi(a), \phi(b)) d u .
$$

Combining (5.5) with (5.6), we get

$$
\mathcal{R}\left(A, \Phi(M), m_{\phi}\right)(a, b)=A\left(\Phi(M)\left(a, m_{\phi}(a, b)\right), \Phi(M)\left(m_{\phi}(a, b), b\right)\right)=\Phi(M)(a, b) .
$$

The proof of the theorem is completed. 
Remark 5.1 If the parameterized mean $\left(m_{t}\right)$ is homogeneous with its associated function $f_{t}$, i.e., $f_{t}(x):=m_{t}(1, x)$, then condition (5.1) can be simplified and is equivalent to

$$
\forall t \in[0,1] \quad f_{t} \circ f_{1 / 2}=f_{t / 2} .
$$

It is easy to see that the parameterized mean of Example 3.4 satisfies the above condition.

Notation In what follows, if $\Phi(x)=x^{p}$ for some real number $p \neq 0$, then we write $\left(m_{1}, m_{2}\right)_{p}$ instead of $\left(m_{1}, m_{2}\right)_{\Phi}$ and $\mathcal{R}_{p}$ instead of $\mathcal{R}_{\Phi}$. For $\Phi(x)=\ln x$, we write $\left(m_{1}, m_{2}\right)_{\ln }$ and $\mathcal{R}_{\ln }$. With Example 4.2, we then have $L$ is $(A, A)_{-1}$-stabilizable, i.e., $\mathcal{R}_{-1}(A, L, A)=L$, and $I$ is $(A, A)_{\ln }$-stabilizable, i.e., $\mathcal{R}_{\ln }(A, I, A)=I$.

Corollary 5.2 Let $p, q$ be two real numbers. Then we have

(1) If $p \neq q$, then $E_{p, q}$ is $\left(A, B_{p}\right)_{q-p}$-stabilizable.

(2) $E_{p, p}$ is $\left(A, B_{p}\right)_{\mathrm{ln}}$-stabilizable.

In particular, for $p \neq 0$ the power logarithmic mean $L_{p}$ is $(A, A)_{p}$-stabilizable.

Proof It is sufficient to combine Theorem 5.1 with Example 3.4. Details are omitted here for the reader.

We end this section by stating the following remark which highlights the interest of the above results.

Remark 5.2 To characterize a mean $M$ as an $(A, m)_{\Phi}$-mean is of great importance since the involved arithmetic mean $A$ is the simplest analytic mean further with a linear affine character. In particular, in the computation context, the relationship

$$
\mathcal{R}\left(A, \sum_{i=1}^{n} \alpha_{i} f_{i}, m\right)=\sum_{i=1}^{n} \alpha_{i} \mathcal{R}\left(A, f_{i}, m\right)
$$

holds for all functions $f_{1}, f_{2}, \ldots, f_{n}$, all means $m$ and every vector $\left(\alpha_{1}, \alpha_{2}, \ldots, \alpha_{n}\right) \in \mathbb{R}^{n}$. See section below for a more explicit explanation.

\section{Application for mean-inequalities}

In this section we present some applications of the above theoretical study for obtaining mean-inequalities. Following Theorem 5.1 and Remark 5.2, we may state the following.

Theorem 6.1 Let $M$ be a $(\Phi, \phi)_{m}$-mean where $\Phi, \phi$ and $m$ are as in Theorem 5.1. Let $\left(m_{i}\right)_{i=1}^{n}$ be n symmetric means such that

$$
\Phi(M) \leq(\geq) \sum_{i=1}^{n} \alpha_{i} \Phi\left(m_{i}\right)
$$

for some probability vector $\left(\alpha_{1}, \alpha_{2}, \ldots, \alpha_{n}\right)$. Then the following holds:

$$
\Phi(M) \leq(\geq) \sum_{i=1}^{n} \alpha_{i} \mathcal{R}\left(A, \Phi\left(m_{i}\right), m_{\phi}\right)
$$


Proof According to Theorem 5.1, the mean $M$ is $\left(A, m_{\phi}\right)_{\Phi}$-stabilizable. This, when combined with relationship (5.7) and Proposition 4.1(ii), yields the desired result after simple manipulation.

Theorem 6.1, when combined with Corollary 5.2, immediately gives the next result.

Corollary 6.2 Let $p \neq 0$ be a fixed real number. Let $\left(m_{i}\right)_{i=1}^{n}$ be $n$ symmetric means such that

$$
L_{p}^{p} \leq(\geq) \sum_{i=1}^{n} \alpha_{i} m_{i}^{p}
$$

for some probability vector $\left(\alpha_{1}, \alpha_{2}, \ldots, \alpha_{n}\right)$. Then we have

$$
L_{p}^{p} \leq(\geq) \sum_{i=1}^{n} \alpha_{i} \mathcal{R}\left(A, m_{i}^{p}, A\right) .
$$

Taking $p=-1$ in the above corollary, with the fact that $L_{-1}=L$, we immediately obtain the next result.

Corollary 6.3 Let $\left(m_{i}\right)_{i=1}^{n}$ be $n$ symmetric means such that

$$
1 / L \leq(\geq) \sum_{i=1}^{n}\left(\alpha_{i} / m_{i}\right)
$$

for some probability vector $\left(\alpha_{1}, \alpha_{2}, \ldots, \alpha_{n}\right)$. Then one has

$$
1 / L \leq(\geq) \sum_{i=1}^{n} \alpha_{i} \mathcal{R}\left(A, 1 / m_{i}, A\right)
$$

Now, we present an example illustrating the above results.

Example 6.1 The inequality

$$
\frac{1}{L} \leq \frac{2}{3} \frac{1}{A}+\frac{1}{3} \frac{1}{H}
$$

is well known in the literature, see [3]. If we apply Corollary 6.3 with $n=2, \alpha_{1}=2 / 3, \alpha_{2}=$ $1 / 3$ and $m_{1}=A, m_{2}=H$, we obtain, after simple computation,

$$
\frac{1}{L} \leq \frac{1}{6} \frac{1}{A}+\frac{1}{6} \frac{1}{H}+\frac{2}{3}\left(\frac{3}{4} A+\frac{1}{4} H\right)^{-1}
$$

Using the convexity of the real-function $x \mapsto 1 / x$ on $(0, \infty)$, the reader can easily verify that the inequality (6.8) refines (6.7), in this way proving the desired aim.

More importance to this example is given by Corollary 6.3. We can apply it again with $n=3, \alpha_{1}=\alpha_{2}=1 / 6, \alpha_{3}=2 / 3$ and $m_{1}=A, m_{2}=H, m_{3}=\frac{3}{4} A+\frac{1}{4} H$. We then obtain

$$
\frac{1}{L} \leq \frac{1}{6} \mathcal{R}(A, 1 / A, A)+\frac{1}{6} \mathcal{R}(A, 1 / H, A)+\frac{2}{3} \mathcal{R}\left(A,\left(\frac{3}{4} A+\frac{1}{4} H\right)^{-1}, A\right) .
$$


We left to the reader the routine task of computing the right-hand side of (6.9), to ensure that the obtained upper bound of $1 / L$ refines that of (6.8) and to show how we can repeat the application of Corollary 6.3 for $n=4$ with convenient means and related coefficients.

Example 6.2 The inequality

$$
\frac{3}{L} \leq \frac{1}{G}+\frac{4}{A+G},
$$

proved in [9] by Neuman and Sándor, is a refinement of (6.7). If we apply Corollary 6.3 to (6.10), with $n=2, \alpha_{1}=1 / 3, \alpha_{2}=2 / 3$ and $m_{1}=G, m_{2}=\frac{A+G}{2}$, we obtain

$$
\frac{1}{L} \leq \frac{1}{3} \mathcal{R}\left(A, \frac{1}{G}, A\right)+\frac{2}{3} \mathcal{R}\left(A, \frac{2}{A+G}, A\right)
$$

This, with the help of Example 4.3 and a simple reduction, yields the following inequality:

$$
\frac{1}{L} \leq \frac{1}{3} \frac{1}{G}\left(\frac{A+G}{2 A}\right)^{1 / 2}+\frac{4}{3} \frac{A+\left(\frac{A^{2}+A G}{2}\right)^{1 / 2}}{\left(\frac{A+G}{2}+\left(\frac{A^{2}+A G}{2}\right)^{1 / 2}\right)^{2}} .
$$

It is easy to verify that this latter inequality refines (6.10), in this way proving the interest of our approach.

\section{Competing interests}

The authors declare that they have no competing interests.

\section{Authors' contributions}

Both authors jointly worked, read and approved the final version of the paper.

\section{Author details}

'Department of Mathematics, Faculty of Science, Taibah University, P.O. Box 30097, Al Madinah Al Munawwarah, 41477, Kingdom of Saudi Arabia. ${ }^{2}$ Department of Mathematics, Science Faculty, Moulay Ismail University, Meknes, Morocco.

\section{Acknowledgements}

The authors thank the anonymous referees for their comments and suggestions which have been included in the final version of this paper. This work was supported by the Research Center of Taibah University 433/1698.

Received: 23 September 2012 Accepted: 20 April 2013 Published: 8 May 2013

\section{References}

1. Raïssouli, M, Sándor, J: On a method of construction of new means with applications. J. Inequal. Appl. 2013,89 (2013)

2. Bullen, PS: Handbook of Means and Their Inequalities. Kluwer, Dordrecht (2003)

3. Chen, C-P: On some inequalities for means and the second Gautschi-Kershaw's inequality. RGMIA 11 Supplement, Art. 6 (2008)

4. Raïssouli, M: Parameterized logarithmic mean. Int. J. Math. Anal. 6(18), 863-869 (2012)

5. Carlson, BC: The logarithmic mean. Am. Math. Mon. 79, 615-618 (1972)

6. Toader, G: Some mean values related to the arithmetic-geometric mean. J. Math. Anal. Appl. 218(2), 358-368 (1998)

7. Toader, G, Sándor, J: Inequalities for general integral means. J. Inequal. Pure Appl. Math. 7(1), Art. 13 (2006)

8. Raïssouli, M: Stability and stabilizability for means. Appl. Math. E-Notes 11, 159-174 (2011)

9. Neuman, E, Sándor, J: On certain means of two arguments and their extensions. Int. J. Math. Math. Sci. 2003(16), 981-993 (2003) 